\title{
Modelling the mass-SFR relation at high redshifts; predicted constraints from JWST
}

\author{
Emma Curtis-Lake \\ KICC, University of Cambridge, United Kingdom
}

\begin{abstract}
The mass-SFR relation of galaxies encodes information of present and historical star formation in the galaxy population. We expect the intrinsic scatter in the relation to increase to low mass where SFR becomes more stochastic. Measurements at $z \gtrsim 4$ from the Hubble Frontier fields have hinted at this (Santini et al., 2017), however, with the added uncertainty of lensing magnification we await JWST to provide robust measurements. Even with datasets provided by JWST, uncertainties on mass and SFR estimates are often large, potentially covariant and dependent on assumptions used. I will present our method of Bayesian hierarchical modelling of the mass-SFR relation that self-consistently propagates uncertainties on mass and SFR estimates to uncertainties on the mass-SFR relation parameters. I will expose the biases imposed by standard SED-modelling practices, and address to what significance we can measure an increase in intrinsic scatter to low masses with JWST.
\end{abstract}

